Open Access

\title{
Unraveling the potential co-contributions of cerebral small vessel vasculopathy to the pathogenesis of Alzheimer's dementia
}

\author{
Jodi D. Edwards ${ }^{1,2^{*}}$, Joel Ramirez ${ }^{1,2}$ and Sandra E. Black ${ }^{1,2,3,4}$ \\ See related research, http://www.alzres.com/content/7/1/27
}

\begin{abstract}
Emerging evidence for the potential co-contributions of small vessel vasculopathy to dementia has resulted in a more nuanced view of Alzheimer's disease (AD) pathogenesis. Although cerebral small vessel disease, visualized on magnetic resonance imaging as hyperintense signal abnormalities, independently predicts the incidence and clinical progression of dementia, the relationships between AD pathology, white matter hyperintensity volume, genotype, and cognitive decline in AD remain unclear. The study by Morgen and colleagues, recently published in Alzheimer's Research \& Therapy, presents important new findings on the associations between apolipoprotien $\mathrm{E} \varepsilon 4$ genotype, white matter hyperintensities, and cognition, independent of vascular risk, in a cohort of AD patients.
\end{abstract}

\begin{abstract}
Alzheimer's disease (AD) is considered the most common pathology contributing to dementia. Although genomic analyses have identified the apolipoprotein $\mathrm{E}$ (apoE) $\varepsilon 4$ allele as a major risk factor for late-onset $\mathrm{AD}$, cerebral small vessel disease (SVD) may also play a significant role in decline to dementia. Despite increased efforts to elucidate the complex relationships between AD, SVD, and apoE genotype, the evidence remains equivocal, posing a challenge for understanding the pathogenic mechanisms underlying aging and dementia.

Prevailing models of $\mathrm{AD}$ pathogenesis suggest that the aggregation of oligomeric amyloid-beta $(\mathrm{A} \beta)$ proteins initiates a pathophysiological cascade hallmarked by extracellular $A \beta$ plaques in the interstitium and along cerebral vessels, and intraneuronal neurofibrillary tangles of hyperphosphorylated tau. Neurodegenerative sequelae include synaptic dysfunction, cortical atrophy, and progressive cognitive decline. In vivo models of $\mathrm{AD}$ pathogenesis have identified several potential mechanisms by which apoE $\varepsilon 4$ may contribute to amyloidogenic processes (Fig. 1) [1].
\end{abstract}

\footnotetext{
* Correspondence: jodi.edwards@sunnybrook.ca

${ }^{1}$ LC Campbell Cognitive Neurology Research Unit, Hurvitz Brain Sciences Research Program, Sunnybrook Research Institute, Toronto, ON M4N 3M5, Canada

${ }^{2}$ Heart \& Stroke Foundation Canadian Partnership for Stroke Recovery, Sunnybrook Health Sciences Centre, Toronto, ON M4N 3M5, Canada Full list of author information is available at the end of the article
}

In recent decades, a more nuanced view of $\mathrm{AD}$ pathogenesis has emerged, with evidence for the putative cocontributions of vascular pathology. SVD is commonly observed at autopsy in patients with $\mathrm{AD}$ and, although neuropathological studies have consistently shown additive, not interactive effects of vasculopathy [2], white matter hyperintensities (WMHs) are more difficult to capture at end-stage disease. Several neuroimaging-derived biomarkers for SVD have been identified, including periventricular and deep WMHs of presumed vascular origin, lacunes, and enlarged perivascular Virchow-Robin spaces, which may be visualized earlier in disease progression [3]. Recent studies using amyloid positron emission tomography (PET) support a relationship between SVD and amyloid pathology [4], but also confirm that significant subcortical ischemic vasculopathy alone may be sufficient to cause dementia [5]. Combined with early clinical evidence for the role of apoE $\varepsilon 4$ in the development of cerebral amyloid angiopathy independent of AD [6], and observational associations between dementia and vascular risk factors, these advances have initiated new lines of investigation into whether white matter vasculopathy represents a synergistic or independent mechanism of $\mathrm{AD}$ pathogenesis.

In a recent article in Alzheimer's Research \& Therapy, Morgen and colleagues [7] retrospectively examined 


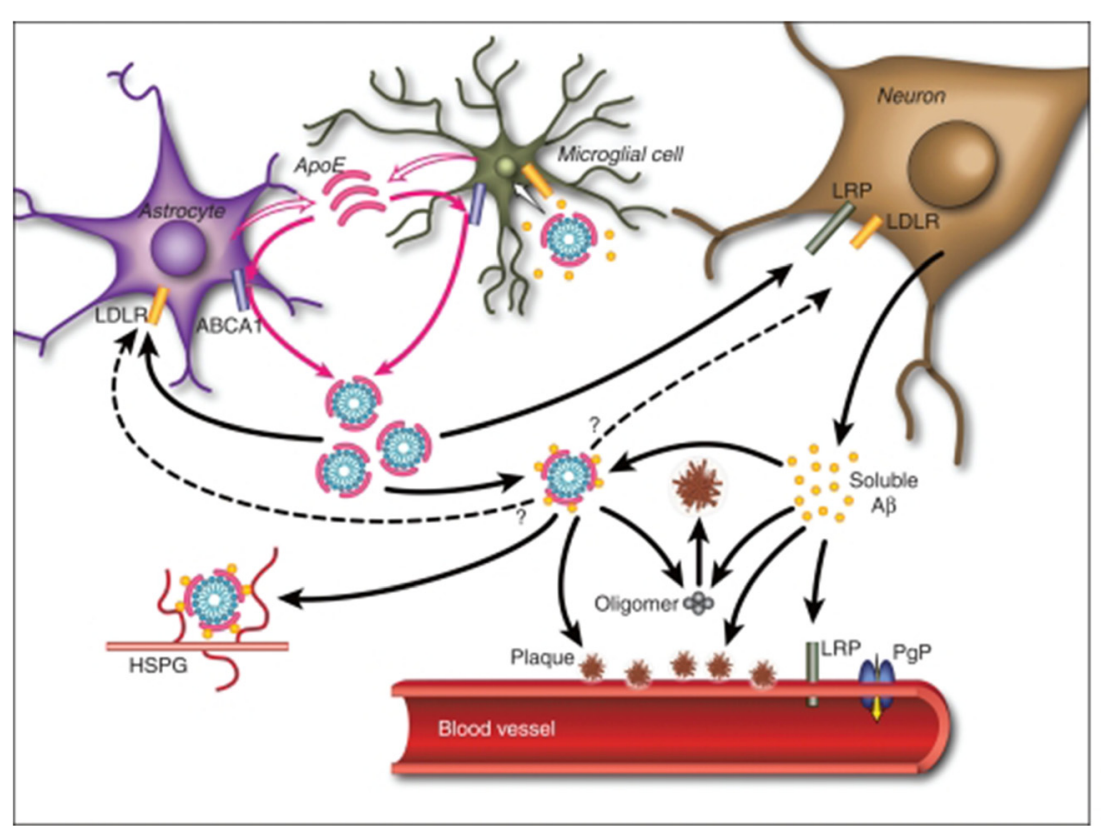

Fig. 1 Effects of apolipoprotein $E(a p o E) \varepsilon 4$ on amyloid-beta (A $\beta$ ) metabolism and deposition. apoE may facilitate cellular uptake of $A \beta$ by the endocytosis of apoE-containing lipoprotein particles bound to soluble $A \beta$ (yellow circles), or via binding of this apoE-A $\beta$ complex (mauve-blue circles) to extracellular heparin sulfate proteoglycans (HSPG). Alternatively, apoE may impair A $\beta$ clearance by slowing the transport of A $\beta$ across the blood-brain barrier [1]. LDLR, low-density lipoprotein receptor; LRP, lipoprotein receptor-related protein; PgP, permeability-glycoprotein. Reproduced with permission from Elsevier [1]

relationships between apoE genotype, WMHs, and cognitive performance in a cohort of patients with mild to moderate $\mathrm{AD}(\mathrm{N}=183)$. They compared apoE $\varepsilon 4$ carriers to non-carriers with respect to global and regional WMH volume and performance on standardized neuropsychological tests. Results indicated that apoE $\varepsilon 4$ carriers showed reduced WMH volumes compared with noncarriers and, among non-carriers, global WMH volume was correlated with performance on the Trail Making Test-A of executive function. The authors interpreted these findings as evidence that WMHs had a functional impact on cognition and that SVD represents an independent mechanism of AD pathogenesis.

The Morgen et al. study is consistent with prior work demonstrating that WMHs primarily impact the domain of executive function [8] and are associated with amyloid plaques on Pittsburg compound B retention PET in apoE $\varepsilon 4$ non-carriers with subcortical vascular cognitive impairment [9]. In a recent meta-analysis of 42 studies, however, an association between apoE $\varepsilon 4$ and magnetic resonance imaging markers of cerebrovascular disease was reported [10], suggesting that vascular mechanisms may differentially co-contribute to multiple pathophysiological cascades with distinct clinical sequelae.

A strength of the Morgen et al. study [7] is in the specificity of case selection. To minimize the potential for misclassification, the authors excluded those with 'severe' WMH burden suggestive of vascular dementia according to new $\mathrm{AD}$ diagnostic guidelines [11]. They then adjusted all multivariate models for multiple vascular risk factors, to increase the likelihood of observing apoE-dependent effects. In restricting their cohort to patients without a presumed vascular etiology, Morgen et al. provide novel evidence for an association between WMH and apoE genotype that modified executive function independent of vascular risk.

A major source of bias in clinical imaging studies is that template-matching approaches, such as statistical parametric mapping, do not include a head size correction or account for cortical atrophy. In many clinical populations, particularly $\mathrm{AD}$, the degree of atrophy is high, impacting ventricle size and total intracranial volume and resulting in the potential misattribution of WMH to the grey matter tissue compartment [12]. To partially address this limitation, Morgen et al. corrected for total intracranial volume and still showed greater WMH burden in apoE $\varepsilon 4$ non-carriers, strengthening their argument that this association reflects pathogenic mechanisms of $\mathrm{AD}$ independent of apoE \&4-mediated neurodegeneration.

As arterial hypertension correlated with WMHs in their cohort, Morgen et al. suggest blood pressure may have synergistic effects on amyloid-mediated endothelial damage. Recent animal studies have described a 'G-lymphatic' system that removes amyloid in the perivascular spaces toward the deep periventricular veins [13] and confirmed 
an association between arterial hypertension and venous collagenosis in spontaneously hypertensive rats [14]. Neuropathological analyses also suggest that periventricular WMHs may represent perivenular vasogenic edema correlating with collagenosis of the deep medullary venules [15]. Venous insufficiency thus represents a potential mechanism of impaired $A \beta$ clearance that may exacerbate amyloid accumulation and increase clinical decline into dementia.

Increasing evidence for the role of small vessel vasculopathy in dementia highlights the importance of multifactorial mechanisms in AD pathogenesis. The findings of Morgen and colleagues are timely and offer support for the co-contributions of WMHs to cognitive impairment in $\mathrm{AD}$, not mediated by apoE $\varepsilon 4$ genotype. A greater understanding of amyloid clearance will be required for the development of targeted therapies to mitigate the risk and clinical progression of AD. Amyloid PET imaging offers new opportunities to elucidate additive/interactive effects of WMHs in AD and unravel the pathogenic mechanisms of this complex phenotype.

\section{Abbreviations}

AD: Alzheimer's disease; apoE: apolipoprotein E; AB: Amyloid-beta; PET: Positron emission tomography; SVD: Cerebral small vessel disease; WMH: White matter hyperintensity.

\section{Competing interests}

The authors declare that they have no competing interests.

\section{Authors' contributions}

JDE drafted the manuscript. JR and SEB reviewed and revised the manuscript draft. All authors read and approved the final manuscript.

\section{Author details}

'LC Campbell Cognitive Neurology Research Unit, Hurvitz Brain Sciences Research Program, Sunnybrook Research Institute, Toronto, ON M4N 3M5, Canada. ${ }^{2}$ Heart \& Stroke Foundation Canadian Partnership for Stroke Recovery, Sunnybrook Health Sciences Centre, Toronto, ON M4N 3M5, Canada. ${ }^{3}$ Institute of Medical Science, University of Toronto, Toronto, ON M5S 1A8, Canada. ${ }^{4}$ Department of Medicine, Neurology, Sunnybrook Health Sciences Centre, Toronto, ON M4N 3M5, Canada.

Published online: 10 July 2015

\section{References}

1. Kim J, Basak JM, Holtzman DM. The role of apolipoprotein E in Alzheimer's disease. Neuron. 2009;63:287-303.

2. Chui HC, Zheng L, Reed BR, Vinters HV, Mack WJ. Vascular risk factors and Alzheimer's disease: are these risk factors for plaques and tangles or for concomitant vascular pathology that increases the likelihood of dementia? An evidence-based review. Alzheimers Res Ther. 2012;4:1.

3. Wardlaw JM, Smith EE, Biessels GJ, Cordonnier C, Fazekas F, Frayne R, et al. Neuroimaging standards for research into small vessel disease and its contribution to ageing and neurodegeneration. Lancet Neurol. 2013;12:822-38.

4. Grimmer T, Faust M, Auer F, Alexopoulos P, Forstl H, Henriksen G, et al. White matter hyperintensities predict amyloid increase in Alzheimer's disease. Neurobiol Aging. 2012;33:2766-73.

5. Kim HJ, Jeon BS, Kim YE, Kim JY, Kim YK, Sohn CH, et al. Clinical and imaging characteristics of dementia in multiple system atrophy. Parkinsonism Related Dis. 2013;19:617-21.

6. Greenberg SM, Rebeck GW, Vonsattel JP, Gomez-Isla T, Hyman BT. Apolipoprotein E epsilon 4 and cerebral hemorrhage associated with amyloid angiopathy. Ann Neurol. 1995;38:254-9.
7. Morgen K, Schneider M, Frölich L, Tost H, Plichta MM, Kölsch H, et al. Apolipoprotein E-dependent load of white matter hyperintensities in Alzheimer's disease: a voxel-based lesion mapping study. Alzheimer's Res Ther. 2015;7:27.

8. Hedden T, Mormino EC, Amariglio RE, Younger AP, Schultz AP, Becker JA, et al. Cognitive profile of amyloid burden and white matter hyperintensities in cognitively normal older adults. J Neurosci. 2012;32:16233-42.

9. Noh Y, Seo SW, Jeon S, Lee JM, Kim JH, Kim GH, et al. White matter hyperintensities are associated with amyloid burden in APOE4 non-carriers. J Alzheimer's Dis. 2014;40:877-86.

10. Schilling S, DeStefano AL, Sachdev PS, Choi SH, Mather KA, DeCarli CD, et al. APOE genotype and MRI markers of cerebrovascular disease: systematic review and meta-analysis. Neurology. 2013;81:292-300.

11. McKhann GM, Knopman DS, Chertkow H, Hyman BT, Jack Jr CR, Kawas CH, et al. The diagnosis of dementia due to Alzheimer's disease: recommendations from the National Institute on Aging-Alzheimer's Association workgroups on diagnostic guidelines for Alzheimer's disease. Alzheimer's Dement. 2011;7:263-9.

12. Ramirez J, McNeely AA, Scott CJ, Stuss DT, Black SE. Subcortical hyperintensity volumetrics in Alzheimer's disease and normal elderly in the Sunnybrook Dementia Study: correlations with atrophy, executive function, mental processing speed, and verbal memory. Alzheimers Res Ther. 2014;6:49.

13. Iliff JJ, Wang M, Liao Y, Plogg BA, Peng W, Gundersen GA, et al. A paravascular pathway facilitates CSF flow through the brain parenchyma and the clearance of interstitial solutes, including amyloid beta. SciTransIMed. 2012;4:147ra111.

14. Zhou M, Mao L, Wang Y, Wang Q, Yang Z, Li S, et al. Morphologic changes of cerebral veins in hypertensive rats: venous collagenosis is associated with hypertension. J Stroke Cerebrovasc Dis. 2014. doi:10.1016/j.jstrokecerebrovasdis.2014.09.038.

15. Black S, Gao F, Bilbao J. Understanding white matter disease: imagingpathological correlations in vascular cognitive impairment. Stroke. 2009;40:S48-52. 